Europhys. Lett., 50 (1), pp. 61-67 (2000)

\title{
Amorphous thin-film growth: Theory compared with experiment
}

\author{
M. Raible, S. G. Mayr $\left(^{*}\right)$, S. J. Linz, M. Moske $\left(^{* *}\right)$, \\ P. HängGI and K. SAmwer $\left(^{*}\right)$ \\ Institut für Physik, Universität Augsburg - D-86135 Augsburg, Germany \\ (received 1 July 1999; accepted in final form 14 January 2000) \\ PACS. 68.35.Bs - Surface structure and topography. \\ PACS. 61.43.Dq - Amorphous semiconductors, metals and alloys.
}

\begin{abstract}
Experimental results on amorphous $\mathrm{ZrAlCu}$ thin-film growth and the dynamics of the surface morphology as predicted from a minimal nonlinear stochastic deposition equation are analysed and compared. Key points of this study are: i) an estimation procedure for coefficients entering into the growth equation and ii) a detailed analysis and interpretation of the time evolution of the correlation length and the surface roughness. The results corroborate the usefulness of the deposition equation as a tool for studying amorphous growth processes.
\end{abstract}

Introduction. - During the last decade the study of the kinetics of surface growth processes has attracted considerable interest (cf. the reviews [1]). The dynamics of the surface morphology, e.g. in amorphous thin-film growth is dominated by the interplay of roughening, smoothening, and pattern forming processes. On the microscopic level, these processes are governed by the highly complex and only partly understood interaction of the depositing particles with the already condensed surface atoms. Despite the complexity of the growth processes on the atomic scale, experiments on the slightly coarser mesoscopic scale typically reveal some sort of regularity of the surface morphology with some superimposed small-scale stochastics $[2,3]$. This, in turn, indicates that the machinery of coarse-grained continuum models based on phenomenologically motivated stochastic growth equations [1] is a useful tool for the understanding and interpretation of the growth dynamics. In particular, amorphous thinfilm growth represents an attractive testing ground for the validation of such phenomenological models; this is mainly due to the spatially isotropic nature of the amorphous structure at this scale and the lack of long-range ordering.

Our objective is a detailed comparison between a stochastic nonlinear evolution equation for amorphous thin-film growth [4] and experimental results on the surface morphology of $\mathrm{ZrAlCu}$ films prepared by physical vapor deposition that are analysed using scanning tunneling microscopy (STM). Using the aforementioned theoretical approach, we develop a method to estimate the phenomenological parameters that is based on the evolution for short times.

(*) Present address: I. Physikalisches Institut, Universität Göttingen - Bunsenstr. 9, D-37073 Göttingen, Germany.

$\left.{ }^{* *}\right)$ Present address: Research center caesar - Friedensplatz 16, D-53111 Bonn, Germany.

(C) EDP Sciences 
vapor particle beam

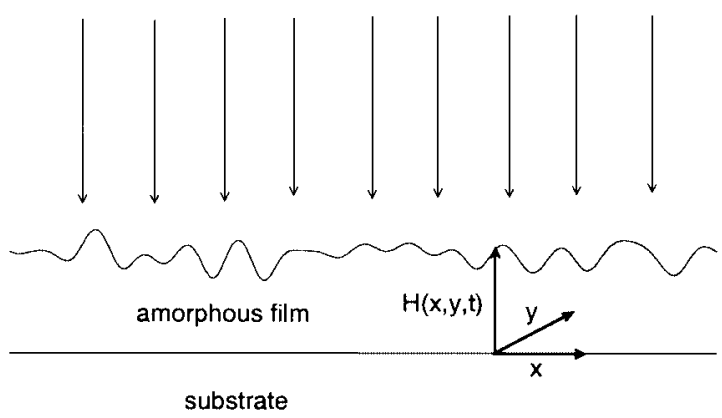

Fig. 1 - Sketch of a physical vapor deposition experiment at normal incidence for amorphous film growth on a substrate.

We also investigate whether the finite apex angle of the cone-like STM tip used in the experiment does affect the comparison between theory and experimental findings. In particular, we demonstrate that the results for both the surface roughness $w$ (i.e. the root-mean-square deviation of the relative height fluctuations) and the correlation length $R_{\mathrm{c}}$ (i.e. the typical length scale over which height fluctuations are correlated) of our full nonlinear growth equation yield very good agreement with the presented experimental data, with only minor modifications due to the finite apex angle. This clearly corroborates the usefulness of our modeling approach.

Experiments. - The glassy $\mathrm{ZrAlCu}$ films (composition $\mathrm{Zr}_{65} \mathrm{Al}_{7.5} \mathrm{Cu}_{27.5}$ ) are prepared in ultra high vacuum by physical vapor deposition on oxidized Si wafers using a total deposition rate of $F=0.79 \mathrm{~nm} / \mathrm{s}$ (electron beam evaporation of the pure elements, each source independently rate controlled). Due to the geometrical arrangements, the particle flux is almost perpendicular to the substrate which is rotated during deposition (for a sketch of the experiment setup cf. fig. 1). The surface profiles of the films (sample area $\left.(200 \times 200) \mathrm{nm}^{2}\right)$ are analysed in situ by scanning tunneling microscopy using a tungsten tip with an apex angle of ca. $40^{\circ}$ (for further details cf. ref. [2]). The film composition is verified by Auger electron spectroscopy. Additionally performed X-ray diffraction and differential scanning calorimetry (DSC) measurements ensure the amorphicity of the investigated films. From the STM data, the correlation length $R_{\mathrm{c}}$ and the surface roughness $w$ (for their definition, see below) are determined for various layer thicknesses up to $480 \mathrm{~nm}$. The experimental results correspond to the diamond symbols depicted in figs. 2 and 3 below. At large layer thicknesses $(\geq 100 \mathrm{~nm})$ each such symbol represents a measurement with a different sample.

Theoretical modeling. - To accomplish a theoretical description of the time evolution of an initially flat surface $H(\vec{x}, t)$, where $H$ denotes the $z$-coordinate of the growing surface at the position $\vec{x}=(x, y)$ and time $t$ (cf. also fig. 1 ), we take advantage of the well-established phenomenological approach that is based on stochastic nonlinear partial differential equations [1], i.e.

$$
\partial_{t} H=G(H, \nabla H)+F+\eta
$$

In eq. (1), $G$ denotes a functional of the surface height and its local derivatives. The detailed functional form of $G$ depends crucially on the considered experimental setup and the kinetics of the deposition process. $F$ denotes the mean deposition rate and $\eta(\vec{x}, t)$ is the related deposition noise that determines the fluctuations of the deposition process around its mean $F$. These 
fluctuations are assumed to be Gaussian white,

$$
\langle\eta(\vec{x}, t)\rangle=0 ; \quad\left\langle\eta(\vec{x}, t) \eta\left(\vec{y}, t^{\prime}\right)\right\rangle=2 D \delta^{2}(\vec{x}-\vec{y}) \delta\left(t-t^{\prime}\right)
$$

where the brackets denote ensemble averaging. It proves useful to introduce the height profile $h(\vec{x}, t)=H(\vec{x}, t)-F t$. If the deposition process has any excess velocity, there is a nonlinear relation between the mean growth or layer thickness $\langle H\rangle$ and time, i.e. $\langle H\rangle(t)=F t+\langle h\rangle(t)$ with $\langle h\rangle(t) \neq 0$.

The simplest nonlinear amorphous thin-film growth equation of the functional form (1) that incorporates i) the physical symmetries such as rotation and reflection invariance in the plane perpendicular to the growth direction, cf. fig. 1, ii) no particle desorption, and iii) the potentiality of local density variations in the amorphously grown material yields, in terms of a low-order expansion of $G$ in the gradients of the surface profile $h(\vec{x}, t)$, the result

$$
\partial_{t} h=a_{1} \nabla^{2} h+a_{2} \nabla^{4} h+a_{3} \nabla^{2}(\nabla h)^{2}+a_{4}(\nabla h)^{2}+a_{5} \operatorname{det}\left(\begin{array}{cc}
\partial_{x}^{2} h & \partial_{y} \partial_{x} h \\
\partial_{x} \partial_{y} h & \partial_{y}^{2} h
\end{array}\right)+\eta,
$$

with $a_{i}(i=1, \ldots, 5)$ being scalar material-dependent coefficients. In eq. (3), the first and the fifth term on the r.h.s. are directly related to the deflection of the initially perpendicular incident particles due to the interatomic forces between the surface atoms and the incident particles. For an indication of the relevance of this effect we refer to the recent experimental study in [5]. The coefficients $a_{1}$ and $a_{5}$ are determined by the relations $a_{1}=-F b$ and $a_{5}=F b^{2}$, where $b$ is the difference between the typical range of the interatomic forces and the equilibrium distance of the adatoms to the surface. Because $b$ is small for the aforementioned experimental setup (typically of the order $10^{-1} \mathrm{~nm}$ ) the term proportional to $a_{5}$ in eq. (3) can safely be neglected. The second and the third term on the r.h.s. of eq. (3) are related to the known microscopic mechanisms of i) the surface diffusion suggested by Mullins [6] and ii) the equilibration of the inhomogeneous concentration of the diffusing particles on the surface, as suggested in $[7,8]$. Moreover, the coefficients $a_{2}$ and $a_{3}$ are negative. The coefficient $a_{3}$ reads $a_{3}=-F l^{2} / 8$, where $l^{2}$ is the mean square of the diffusion length of the particles. This characteristic dependence for amorphous growth is similar in nature to a term appearing for crystalline growth [9]; it differs, however, due to the absence of an additional length scale, the typical height of crystalline terraces. The fourth term on the r.h.s. of eq. (3) is of KardarParisi-Zhang (KPZ) form [10]. It is due to the potential dependence of the local density on the surface slope $\nabla h$, i.e. $[\rho(\nabla h)]^{-1}=\rho_{0}^{-1}\left[1+\left(a_{4} / F\right)(\nabla h)^{2}+\mathcal{O}\left((\nabla h)^{4}\right)\right]$, with $a_{4}$ being necessarily positive because of the additional volume increase caused by oblique particle incidence. Note also that a finite $a_{4}$ results in a finite excess velocity.

The experimentally detected correlation length $R_{\mathrm{c}}(t)$ and surface roughness $w(t)$ are determined by the height-height correlation function

$$
C(r, t)=\left.\left\langle\frac{1}{L^{2}} \int \mathrm{d}^{2} x(h(\vec{x}, t)-\bar{h})(h(\vec{x}+\vec{r}, t)-\bar{h})\right\rangle\right|_{|\vec{r}|=r},
$$

where $\bar{h}$ is the spatial average of the surface profile $\bar{h}=\frac{1}{L^{2}} \int \mathrm{d}^{2} y h(\vec{y}, t)$ and $L^{2}$ the sample area. Specifically, $R_{\mathrm{c}}(t)$ is given by the first maximum of $C(r, t)$ occurring at nonzero $r$ and the square of the surface roughness results by taking the limit $r=0$ in $C(r, t)$, i.e. $w^{2}(t)=C(0, t)$. To integrate numerically the growth equation, a forward-backward finite difference method on a quadratic lattice combined with an Euler algorithm in time and periodic boundary conditions on a quadratic area $[0, L]^{2}$ have been invoked [11]. 

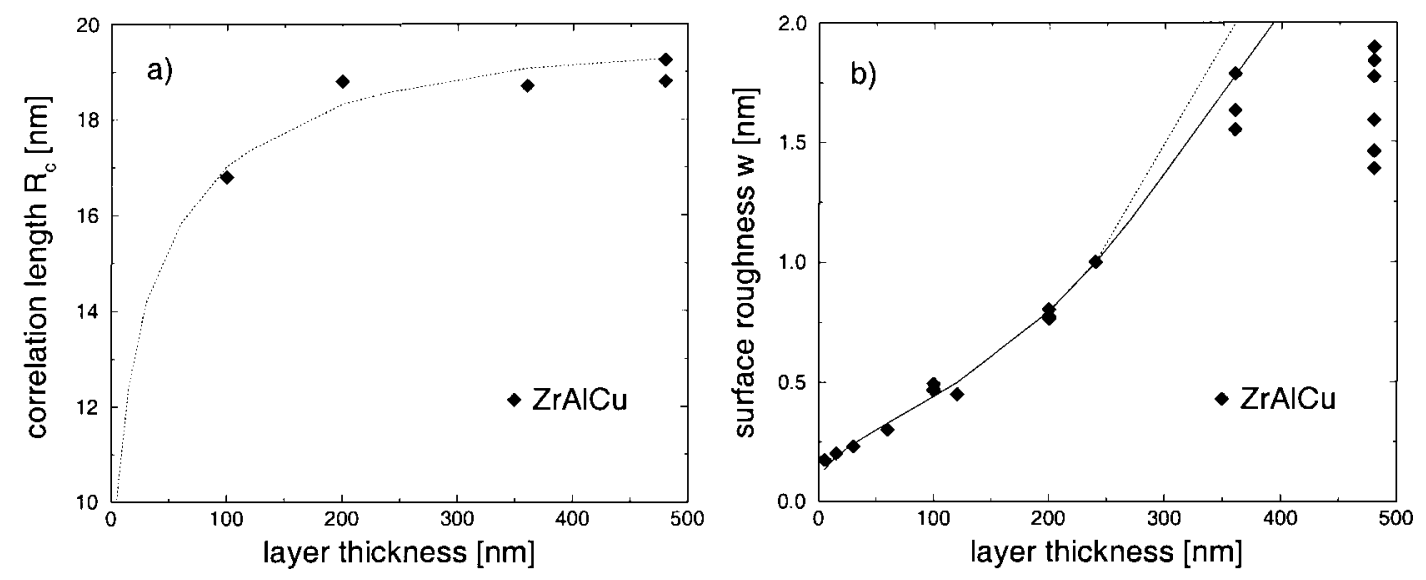

Fig. 2 - Correlation length $R_{\mathrm{c}}$ and surface roughness $w$ (dotted lines without consideration of the STM tip), defined below eq. (4), vs. layer thickness $\langle H\rangle$, see below eq. (2), are calculated from the linear growth equation (5) using the parameters given in eq. (7). In panel b we depict the influence of the STM tip with an apex angle of $100^{\circ}$ (solid line). The diamond symbols represent the corresponding experimental results.

Linear model equation. - In order to make contact with the aforementioned experiment, we first extrapolate the values of the parameters $a_{1}, a_{2}$, and $D$. The initial stages of the deposition process (corresponding to layer thicknesses $\langle H\rangle \leq 240 \mathrm{~nm}$ in the experiment), which started from a flat substrate, are dominated by the temporal evolution of the linear limit of eq. (3),

$$
\partial_{t} h=a_{1} \nabla^{2} h+a_{2} \nabla^{4} h+\eta
$$

The growth of the Fourier modes of the surface profile is determined by $\partial_{t} \tilde{h}(\vec{k}, t)=\sigma(k) \tilde{h}(\vec{k}, t)+$ $\tilde{\eta}(\vec{k}, t)$, where $\sigma(k)=-a_{1} k^{2}+a_{2} k^{4}$ denotes the growth rate of the Fourier modes. Therefore, the wave number $k_{\mathrm{c}}$ belonging to the maximum of the growth coefficient $\sigma(k)$ reads $k_{\mathrm{c}}=\sqrt{a_{1} / 2 a_{2}}$. The correlation length $R_{\mathrm{c}}(t)$ of the surface profile $h(\vec{x}, t)$ arising from the linearized equation (5) follows initially a $t^{1 / 4}$-law, whereas it saturates at later stages into $R_{\mathrm{c}}(t)=7.0156 / k_{\mathrm{c}}=7.0156 \sqrt{2 a_{2} / a_{1}}$ when the critical mode with the wave number $k_{\mathrm{c}}$ dominates. Since a saturation of $R_{\mathrm{c}}(t)$ with increasing layer thickness is also observed in the experiment for layer thicknesses larger than $200 \mathrm{~nm}$ (cf. the diamond symbols in fig. 2a), the ratio $a_{2} / a_{1}$ can be roughly estimated. The surface roughness $w(t)$ at later stages follows an $\exp \left[\sigma\left(k_{\mathrm{c}}\right) t\right]=\exp \left[-a_{1}^{2} t / 4 a_{2}\right]$-behaviour when the critical mode dominates. By a comparison with the experimentally observed increase of $w(t)$ (cf. the diamond symbols in fig. 2b) during the time interval when the layer thickness $\langle H\rangle$ is between $30 \mathrm{~nm}$ and $240 \mathrm{~nm}$, the ratio $a_{1}^{2} / a_{2}$ can be estimated. From the ratios $a_{2} / a_{1}$ and $a_{1}^{2} / a_{2}$ the coefficients $a_{1}$ and $a_{2}$ can be deduced. The height-height correlation function $C(r, t)$ that arises from the linearized equation (5) is determined by

$$
C(r, t)=\left.\frac{D}{(2 \pi)^{2}} \int \mathrm{d}^{2} k \exp [i \vec{k} \cdot \vec{r}] \frac{\exp \left[2\left(-a_{1} k^{2}+a_{2} k^{4}\right) t\right]-1}{-a_{1} k^{2}+a_{2} k^{4}}\right|_{|\vec{r}|=r} .
$$

From $C(r, t)$ the surface roughness $w(t)$ and the correlation length $R_{\mathrm{c}}(t)$ can be determined. This makes it possible to determine more precisely the coefficients of eq. (5). The physical dimensions of these coefficients are $\left[a_{1}\right]=\bar{l}^{2} / \bar{t},\left[a_{2}\right]=\bar{l}^{4} / \bar{t}$, and $[D]=\bar{h}^{2} \bar{l}^{2} / \bar{t}$, where $\bar{l}$ denotes 

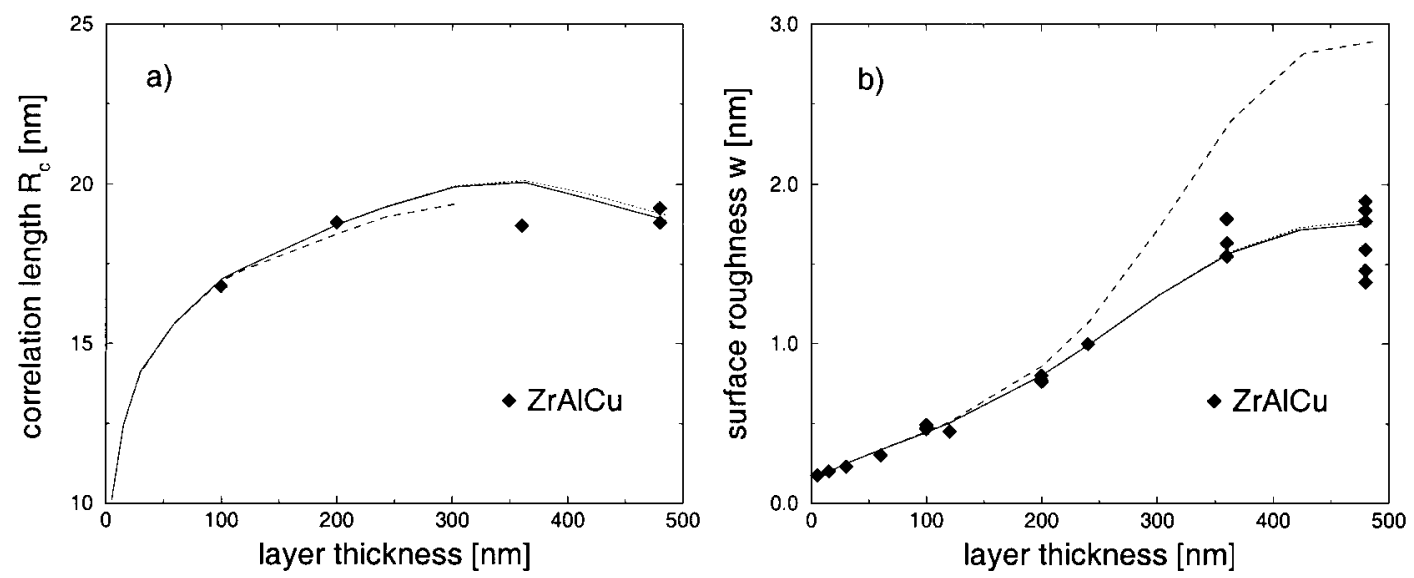

Fig. 3 - Correlation length $R_{\mathrm{c}}$ and surface roughness $w$ calculated from the nonlinear growth equation (8) using the parameters $a_{3}=-0.10 \mathrm{~nm}^{3} / \mathrm{s}$ and $a_{4}=0.055 \mathrm{~nm} / \mathrm{s}$ with (solid lines) and without (dotted lines) consideration of the STM tip (with an apex angle of $100^{\circ}$ ). The parameters of the linear parts of the equation, $a_{1}, a_{2}$, and $D$, are given in eq. (7). To demonstrate the physical relevance of the nonlinear growth law $\propto \nabla^{2}(\nabla h)^{2}$, we depict for comparison the prediction that results by setting $a_{3}=0$ (dashed lines without consideration of the STM tip). The diamond symbols represent the corresponding experimental results.

a length unit, $\bar{t}$ a time unit, and $\bar{h}$ a height unit, respectively. Therefore, $\sqrt{a_{2} / a_{1}}$ is a length constant, $\left|a_{2} / a_{1}^{2}\right|$ is a time constant, and $\sqrt{D /\left|a_{1}\right|}$ is a height constant. Changing $\sqrt{a_{2} / a_{1}}$ by an arbitrary factor would change all lengths by the same factor. So we calculated $w(t)$ and $R_{\mathrm{c}}(t)$ by means of eq. (6) with the approximately determined coefficients $a_{1}$ and $a_{2}$ and an arbitrary $D$. Then, we changed $R_{\mathrm{c}}$, time $t$, and $w$ by independent factors and $\sqrt{a_{2} / a_{1}},\left|a_{2} / a_{1}^{2}\right|$, and $\sqrt{D /\left|a_{1}\right|}$ by the same factors, respectively, until $R_{\mathrm{c}}(t)$ and $w(t)$ were in accordance with the experimental result. By means of $\sqrt{a_{2} / a_{1}},\left|a_{2} / a_{1}^{2}\right|$, and $\sqrt{D /\left|a_{1}\right|}$ we evaluated $a_{1}, a_{2}$, and $D$. The parameters that fit the experimental results best are found to read

$$
a_{1}=-0.0826 \mathrm{~nm}^{2} / \mathrm{s}, \quad a_{2}=-0.319 \mathrm{~nm}^{4} / \mathrm{s}, \quad D=0.0174 \mathrm{~nm}^{4} / \mathrm{s} .
$$

The dotted lines in fig. $2 \mathrm{a}$ and $\mathrm{b}$ depict the corresponding numerical simulations of eq. (5). There is obviously good agreement with the experimental results for layer thicknesses $\langle H\rangle \leq$ $240 \mathrm{~nm}$.

Next, we investigate possible effects caused by the STM tip. Although the detailed shape of the STM tip is known only roughly, a reasonable model, which refers to the worst case situation, consists of a combination of a taper shank with an apex angle of ca. $40^{\circ}$ and a top cone having a considerably wider apex angle of approximately $\alpha=100^{\circ}$. Only the latter part is of relevance for the detection of the surface profile. The effect of the STM tip is that it maps the height profile $h(\vec{x}, t)$ from eq. (5) on the "scanned" height profile $\hat{h}(\vec{x}, t)$. The solid line in fig. $2 \mathrm{~b}$ depicts the corresponding finding for this worst case of a STM tip with an apex angle of $100^{\circ}$. The major effect of the finite tip angle results in a slow down of the growth of the surface roughness $w(t)$. This is caused by the scanning procedure that cannot fully resolve the grooves of the height profile $h(\vec{x}, t)$. As can be deduced from fig. $2 \mathrm{~b}$, however, the linear growth equation (5) (even including the tip angle) is not sufficient to describe the later stages of amorphous thin-film growth. 
Nonlinear model equation. - We next investigate the physical relevance of the nonlinear terms $a_{3} \nabla^{2}(\nabla h)^{2}$ and $a_{4}(\nabla h)^{2}$ on the growth dynamics, i.e. we analyse the nonlinear growth model

$$
\partial_{t} h=a_{1} \nabla^{2} h+a_{2} \nabla^{4} h+a_{3} \nabla^{2}(\nabla h)^{2}+a_{4}(\nabla h)^{2}+\eta .
$$

The parameter choice that fits the experimental results best is found to read

$$
a_{3}=-0.10 \mathrm{~nm}^{3} / \mathrm{s} \text { and } a_{4}=0.055 \mathrm{~nm} / \mathrm{s},
$$

where the parameters given in eq. (7) were used. The corresponding results for the correlation length $R_{\mathrm{c}}(t)$ and the surface roughness $w(t)$ of eq. (8) are depicted in fig. 3 with (solid lines) and without (dotted lines) the inclusion of the effect of the STM tip. We now obtain good agreement with the experimental data also at larger layer thicknesses. Moreover, the finite apex angle of the tip yields only a minor effect (up to the largest experimental layer thickness $\approx 480 \mathrm{~nm}$ ), see the barely visible dotted line in fig. 3b. As a general consequence, the two nonlinear terms result in a drastic slow down of the increase of the surface roughness $w(t)$ with time or layer thickness. The larger the absolute values of $a_{3}$ and $a_{4}$ are, the stronger this slow down is. Nevertheless, the inclusion of both nonlinearities is necessary to obtain a consistent agreement with the experimental data.

In particular, by setting $a_{4}=0$ we need to decrease further $a_{3}$ in order to fit reasonably well the data. Nevertheless, the surface roughness $w(t)$ is now either too large at large thickness $\langle H\rangle=480 \mathrm{~nm}$, or too small at $\langle H\rangle=360 \mathrm{~nm}$ (not shown). The inclusion of the finite tip geometry does not cure this finding. Omitting $a_{3}$ we find an overshoot for the roughness $w(t)$ above $\langle H\rangle \approx 240 \mathrm{~nm}$. In addition, we note that the correlation length $R_{\mathrm{c}}(t)$ ceases to exist above $\langle H\rangle \approx 300 \mathrm{~nm}$, because the first maximum of the height-height correlation $C(r, t)$ vanishes. These features are depicted by the dashed lines in fig. 3a and b. Figure 3b also shows that the experimental data for $w(t)$ are strongly scattered at largest layer thickness. Thus, a precise estimation of the nonlinear coefficients is difficult. We estimate that the coefficient $a_{3}$ ranges at most between $-0.13 \mathrm{~nm}^{3} / \mathrm{s} \leq a_{3} \leq-0.08 \mathrm{~nm}^{3} / \mathrm{s}$ and likewise $0.04 \mathrm{~nm} / \mathrm{s} \leq a_{4} \leq$ $0.07 \mathrm{~nm} / \mathrm{s}$. Consistent with the fitting procedure in fig. $2 \mathrm{a}$ and $\mathrm{b}$, we find that the crossover from linear to nonlinear growth behaviour sets in at $\langle H\rangle \approx 240 \mathrm{~nm}$. An inclusion of the small correction proportional to $a_{5}$, see eq. (3), does not quantitatively impact our results: For the largest layer thickness we find an improvement of maximal $1.0 \%$ for the roughness $w(t)$ and $2.5 \%$ for the correlation length $R_{\mathrm{c}}(t)$.

Discussion. - The aforementioned extrapolation of the parameters $a_{1}, a_{2}, a_{3}, a_{4}$, and $D$ also allows for additional microscopic estimates. i) Since $a_{1}=-F b$, the typical range $b$ of the interaction between the surface atoms and the particles to be deposited is about $0.1 \mathrm{~nm}$, i.e. it approximately equals the size of the radii $(0.2 \mathrm{~nm})$ of the surface atoms. ii) Since $a_{3}=-F l^{2} / 8$, the diffusion length $l$ must be in the range of $1.0 \mathrm{~nm}$. This substantiates that the deposited particles experience a surface diffusion on a nanometer scale and do not just stick at the places where they hit the surface. iii) If the particles arrive independently on the surface, the deposition noise is related to the particle volume $\Omega$ and the mean deposition rate $F$ by $2 D=F \Omega$, yielding (by use of eq. (7)) $\Omega=0.04 \mathrm{~nm}^{3}$. This agrees within a factor of two with the averaged particle volume of $\mathrm{ZrAlCu}$. iv) The necessity of the inclusion of the term proportional to $a_{4}$ indicates that the local density of the growing film varies with the surface slope: On an inclined surface area the local density is decreased by $\rho(\nabla h)=\rho_{0} / \gamma$ with $\gamma=1+\left(a_{4} / F\right)(\nabla h)^{2}$, where $a_{4} / F$ is in the range of 0.07 . Note that these predicted finite density variations are physically compatible with the small diffusion length $l$ of two to three atom diameters. At largest layer thickness $(\approx 480 \mathrm{~nm})$ this local density reduction (averaged 
over the surface) possesses a mean $\bar{\gamma}=1.021$ and a standard deviation $\left(\overline{(\gamma-\bar{\gamma})^{2}}\right)^{1 / 2}=0.017$. The maximum of $\gamma$ on the same surface (at $L=200 \mathrm{~nm}$ ) is typically of the order of 1.12 . At the same time, $\gamma$ averaged over the whole film equals $\langle H\rangle /(F t)=1.01$.

Conclusions. - Starting from experimental data for amorphous $\mathrm{ZrAlCu}$ thin-film growth, the phenomenological growth equation in (3), and using a step-by-step parameter identification procedure, we have shown that a quantitative agreement between theory and experiment is achieved. Based on our comparison between theory and experiment, we conclude that i) eq. (3) constitutes a valid theoretical model for amorphous thin-film growth (at least up to the considered layer thicknesses) and ii) that an interpretation of the data necessarily requires the inclusion of the two nonlinear terms $\nabla^{2}(\nabla h)^{2}$ and $(\nabla h)^{2}$ in the stochastic growth equation (3). Several questions, however, remain open for future studies: a) From the available experimental data it is not clear whether the correlation length $R_{\mathrm{c}}(t)$ and the surface roughness $w(t)$ saturate for larger layer thicknesses. b) The validity of the proposed approach needs to be tested more thoroughly by direct comparison with experimental data for spatio-temporal quantities such as the height-height correlation function. c) A further experimental challenge presents the validation of the theoretically predicted local density variations.

This work has been supported by the DFG-Sonderforschungsbereich 438 München/ Augsburg, TP A1. The authors are indebted to B. REINKER for providing some additional experimental results. The authors like to thank Prof. D. E. WOLF for his most helpful and constructive remarks.

\section{REFERENCES}

[1] Barabasi A. L. and Stanley H. E., Fractal Concepts in Surface Growth (Cambridge University Press) 1995; Tong W. M. and Williams R. S., Annu. Rev. Phys. Chem., 45 (1994) 401; Krug J., Adv. Phys., 46 (1997) 139; Marsili M., Maritan A., Toigo F. and Banavar J. R., Rev. Mod. Phys., 68 (1996) 963.

[2] Reinker B., Moske M. and Samwer K., Phys. Rev. B, 56 (1997) 9887; Mayr S. G., Moske M. and Samwer K., Europhys. Lett., 44 (1998) 465; Mayr S. G., Moske M., and Samwer K., Phys. Rev. B, 60 (1999) 16950; MAYR S. G., Oberflächenrauhigkeit von amorphen ZrAlCuSchichten, Diplomarbeit, Universität Augsburg, 1997.

[3] Salditt T., Metzger T. H., Peisl J., Reinker B., Moske M. and Samwer K., Europhys. Lett., 32 (1995) 331.

[4] Raible M., Linz S. J., and HäNGGi P., unpublished.

[5] van Dijken S., Jorritsma L. C. and Poelsema B., Phys. Rev. Lett., 82 (1999) 4038.

[6] Mullins W. W., J. Appl. Phys., 28 (1957) 333; Wolf D. E. and Villain J., Europhys. Lett., 13 (1990) 389; Villain J., J. Phys. I, 1 (1991) 19; Das Sarma S. and Tamborenea P., Phys. Rev. Lett., 66 (1991) 325; Lai Z.-W. and Das Sarma S., Phys. Rev. Lett., 66 (1991) 2348.

[7] Villain J., J. Phys. I, 1 (1991) 19.

[8] Moske M., Mechanische Spannungen als Sonde für Schichtwachstum und Schichtreaktionen, Habilitationsschrift, Universität Augsburg, 1997; Moske M., Influence of surface topography and roughness evolution on the mechanical stresses in amorphous thin films, submitted for publication.

[9] Kallabis H., Brendel L., Krug J. and Wolf D. E., Int. J. Mod. Phys. B, 11 (1997) 3621.

[10] Kardar M., Parisi G. and Zhang Y.-C., Phys. Rev. Lett., 56 (1986) 889.

[11] Moser K., Kertész J. and Wolf D. E., Physica A, 178 (1991) 215; Amar J. G. and Family F., Phys. Rev. A, 41 (1990) 3399; Lam C.-H. and Shin F. G., Phys. Rev. E, 58 (1998) 5592. 